\title{
Translating Cardiovascular Genomics to Clinical Practice
}

\section{Y. Eugene Chen ${ }^{1,2,3}$}

Accepted: 19 March 2021 / Published online: 14 April 2021

(C) Springer Science+Business Media, LLC, part of Springer Nature 2021
Cardiovascular diseases (CVDs) and stroke are the leading cause of morbidity and mortality in the USA and worldwide [1]. Decades of studies uncovered essential risk factors contributing to CVDs including behavioral and health factors. Nonetheless, and in spite of advancements in diagnosis and treatment, the burden of CVDs and stroke measured as lives lost, as a driver of disabilities and health disparities, and through direct and indirect costs continue on a steep increase. Therefore, understanding the genetic individual risk can contribute substantially to the global fight against CVD at the individual level through novel approaches in personalized medicine that go beyond the modifiable risk factors.

Genome-wide association studies (GWAS) were made possible from the revolution brought about by the sequencing of the human genome in 2000. New sequencing technologies and assembly algorithms for rapid turnaround times reduced costs, and ever-improving analysis tools saw the short period spanning 2005-2007, as the herald for studies that went from associations at the chromosome level [2-4] to the development and exponential growth of GWAS, thus allowing the study of genetic mutations and individual variants at the loci level and their association with CVD. Pioneering studies are illustrated by the identification of SNPs conferring risk of myocardial infarction and their functional analysis by Ozaki and Tanaka [5] in 2005, and the virtually simultaneous publication of the works by Winkelmann et al. [6] and Stefansson

This article belongs to the Topical Collection: Translating genome medicine to treatments

Y. Eugene Chen

echenum@umich.edu

1 Department of Internal Medicine, University of Michigan Medical Center, 2800 Plymouth Rd, NCRC-26 Rm 361S, Ann Arbor, MI 48109-2800, USA

2 Department of Cardiac Surgery, University of Michigan Medical Center, Ann Arbor, MI 48109, USA

3 Center for Advanced Models for Translational Sciences and Therapeutics, University of Michigan Medical Center, Ann Arbor, MI 48109, USA et al. [7] in 2007 on restless leg syndrome, a neurological disorder associated with increased CVD morbidity, identifying different loci and underscoring some of the limitations of those early studies. A GWAS study for CVDs published in 2007 [8-10] identified the association of variants in chromosome 9p21.3 and CVDs. This region contains the genes CDKN2A, CDKN2B, MTAP, and several non-coding RNAs. Thirteen years later, this region continues to be actively investigated [11] to further understand how variation in 9p21.3 might influence hypertension components of cardiovascular risk and further dissect the causal genes. Increased accessibility to data through public repositories, the simultaneous advent of Consortia involving ever-increasing numbers of patients in multiple sites together with the assembly of large teams of researchers with complementary expertise, further propelled the GWAS into an exponential growth as the tool of choice to identify causative/risk SNPs with associated functional studies. Early studies, all in 2007, illustrating this stage include the seminal work of the Welcome Trust Consortium involving the genome-wide association study of 14,000 cases of seven common diseases and 3000 shared controls [12]. The highly publicized international study identified genetic variants in the FTO gene (encoding the alpha-ketoglutaratedependent dioxygenase) associated with obesity-related traits in 6148 individuals from a relatively isolated population in a cluster of four towns in Sardinia, Italy [13]. And finally, the publication of 100K SNP genome-wide association study as a resource from the Framingham Heart Study (FHS), leveraging the most comprehensively characterized multi-generational study at the time, which had been started in 1948 to examine the epidemiology of cardiovascular disease [14], together with the ancillary FHS study on diabetes and related traits integrating different data sets [15]. Since then, GWAS have benefited from the increased number of publicly accessible databases and the creation and sustainability of several large GWAS consortia, which in the cardiovascular field include the Coronary ARtery DIsease Genome-Wide Replication And Meta-Analysis (CARDIoGRAM) [16], the Myocardial Infarction Genetics Consortium (MIGen) [17], Coronary 
Artery Disease (C4D) Genetics Consortium [18], UK biobank [18], and the Million Veteran Program [19].

The increase in population size, arising in part from the increased public awareness, acceptance, and involvement in GWAS studies, together with the improvement in sequencing technologies, data handling, and analysis continues to reveal more candidate genes as causal for CVDs [20], as it is reviewed in detail by Surakka et al. in this issue, with a focus on genomics studies on lipid metabolism as a driver of coronary artery disease [21]. More recently, those advances allowed for the unraveling of the interaction between loci in this group of complex diseases towards the prediction of genetic predisposition at the individual level to begin, which opens new translational opportunities, as it is discussed by Arnold et al. in this issue with a focus on atherosclerotic cardiovascular disease [22].

Although GWAS can inform the involvement in CVDs of unsuspected signaling pathways, they cannot provide detailed mechanistic insight. In fact, the use of enabling technologies, increasingly used in tandem with GWAS within integrated multidisciplinary platforms, has allowed to address fundamental mechanistic and physiologic aspects of both newly identified causal genes as well as the effects of specific variants, whether in the coding or in regulatory regions alike. Lu et al. offer an overview of the impact of these enabling technologies that provide mechanistic input to GWAS findings and highlight the opportunities from emerging integration of multi-omics approaches [23]. Leverage of these technologies is essential for genes that have no homologs in other species, as exemplified by the increasingly recognized role of IncRNAs in CVDs, as discussed in the review by Xiangbo Ruan [24].

Finally, GWAS ultimate goal is to facilitate the development and implementation of personalized medicine. Magavern et al. review the increasing role of pharmacogenomics in cardiovascular medicine and how it can inform clinical practice towards achieving the next frontier of personalized pharmacogenomics [25]. An important example of pharmacogenomics in cardiovascular medicine is illustrated by current efforts towards addressing significant residual cardiovascular risk still remaining, in spite of the effectiveness of statins in reducing LDL-c, which is mostly associated to lingering high triglyceride levels. In that regard, Shaik et al. close this review series by discussing how the association between triglycerides and the genetic risk of atherosclerotic cardiovascular disease can guide pharmacotherapies to reduce the levels of triglycerides and triglyceride-rich lipoproteins towards further reducing risk of CVD [26].

In summary, insight from GWAS continues to uncover candidate genes for intervention while integration of the findings in polygenic risk scores may further help inform clinical practice by identifying individuals at high risk and implementing personalized preventive approaches to minimize risk or utilizing tailored pharmacotherapies, with the ultimate goal of reducing the individual and societal burden of cardiovascular disease-associated disabilities and death.

\section{References}

1. Virani SS, Alonso A, Benjamin EJ, Bittencourt MS, Callaway CW, Carson AP, et al. Heart disease and stroke statistics\&\#x2014;2020 update: a report from the American Heart Association. Circulation. 2020;141(9):e139-596.

2. Shearman AM, Ordovas JM, Cupples LA, Schaefer EJ, Harmon MD, Shao Y, et al. Evidence for a gene influencing the TG/HDL-C ratio on chromosome 7q32.3-qter: a genome-wide scan in the Framingham study. Hum Mol Genet. 2000;9(9):1315-20.

3. Elbein SC, Hasstedt SJ. Quantitative trait linkage analysis of lipidrelated traits in familial type 2 diabetes: evidence for linkage of triglyceride levels to chromosome 19q. Diabetes. 2002;51(2):528 35 .

4. Malhotra A, Wolford JK, American Diabetes GSG. Association, Analysis of quantitative lipid traits in the genetics of NIDDM (GENNID) study. Diabetes. 2005;54(10):3007-14.

5. Ozaki K, Tanaka T. Genome-wide association study to identify SNPs conferring risk of myocardial infarction and their functional analyses. Cell Mol Life Sci. 2005;62(16):1804-13.

6. Winkelmann J, Schormair B, Lichtner P, Ripke S, Xiong L, Jalilzadeh S, et al. Genome-wide association study of restless legs syndrome identifies common variants in three genomic regions. Nat Genet. 2007;39(8):1000-6.

7. Stefansson H, Rye DB, Hicks A, Petursson H, Ingason A, Thorgeirsson TE, et al. A genetic risk factor for periodic limb movements in sleep. N Engl J Med. 2007;357(7):639-47.

8. McPherson R, Pertsemlidis A, Kavaslar N, Stewart A, Roberts R, Cox DR, et al. A common allele on chromosome 9 associated with coronary heart disease. Science. 2007;316(5830):1488-91.

9. Helgadottir A, Thorleifsson G, Manolescu A, Gretarsdottir S, Blondal T, Jonasdottir A, et al. A common variant on chromosome 9 p21 affects the risk of myocardial infarction. Science. 2007;316(5830):1491-3.

10. Genome-wide association study of 14,000 cases of seven common diseases and 3,000 shared controls. Nature. 2007;447(7145):66178.

11. Gallo JE, Ochoa JE, Warren HR, Misas E, Correa MM, GalloVillegas JA, et al. Hypertension and the roles of the 9p21.3 risk locus: classic findings and new association data. Int J Cardiol Hypertens. 2020;7:100050.

12. Wellcome Trust Case Control, C. Genome-wide association study of 14,000 cases of seven common diseases and 3,000 shared controls. Nature. 2007;447(7145):661-78.

13. Scuteri A, Sanna S, Chen WM, Uda M, Albai G, Strait J, et al. Genome-wide association scan shows genetic variants in the FTO gene are associated with obesity-related traits. PLoS Genet. 2007:3(7):e115.

14. Cupples LA, et al. The Framingham Heart Study 100K SNP genome-wide association study resource: overview of 17 phenotype working group reports. BMC Med Genet. 2007;8(Suppl 1):S1.

15. Florez JC, Manning AK, Dupuis J, McAteer J, Irenze K, Gianniny $\mathrm{L}$, et al. A $100 \mathrm{~K}$ genome-wide association scan for diabetes and related traits in the Framingham Heart Study: replication and integration with other genome-wide datasets. Diabetes. 2007;56(12): 3063-74.

16. Preuss M, König IR, Thompson JR, Erdmann J, Absher D, Assimes TL, et al. Design of the Coronary ARtery DIsease Genome-Wide Replication And Meta-Analysis (CARDIoGRAM) Study: a 
genome-wide association meta-analysis involving more than 22 000 cases and 60000 controls. Circ Cardiovasc Genet. 2010;3(5): 475-83.

17. Myocardial Infarction Genetics, $\mathrm{C}$, et al. Genome-wide association of early-onset myocardial infarction with single nucleotide polymorphisms and copy number variants. Nat Genet. 2009;41(3): 334-41.

18. Coronary Artery Disease Genetics, C. A genome-wide association study in Europeans and South Asians identifies five new loci for coronary artery disease. Nat Genet. 2011;43(4):339-44.

19. Evangelou E, et al. Genetic analysis of over 1 million people identifies 535 new loci associated with blood pressure traits. Nat Genet. 2018;50(10):1412-25.

20. Erdmann J, et al. A decade of genome-wide association studies for coronary artery disease: the challenges ahead. Cardiovasc Res. 2018;114(9):1241-57.

21. Crone B, Krause AM, Hornsby WE, Willer CJ, Surakka I. Translating genetic association of lipid levels for biological and clinical application. Cardiovasc Drugs Ther. 2021.
22. Arnold N, Koenig W. Polygenic Risk Score: Clinically Useful Tool for Prediction of Cardiovascular Disease and Benefit from LipidLowering Therapy? Cardiovasc Drugs Ther. 2020.

23. $\mathrm{Lu} \mathrm{H}$, et al. Integration of transformative platforms for the discovery of causative genes in cardiovascular diseases. Cardiovasc Drugs Ther. 2021.

24. Ruan X. Targeting Human lncRNAs for Treating Cardiometabolic Diseases. Cardiovasc Drugs Ther. 2021.

25. Magavern EF, Kaski JC, Turner RM, Janmohamed A, Borry P, Pirmohamed M. The Interface of Therapeutics and Genomics in Cardiovascular Medicine. Cardiovasc Drugs Ther. 2021.

26. Shaik A, Rosenson RS. Genetics of Triglyceride-Rich Lipoproteins Guide Identification of Pharmacotherapy for Cardiovascular Risk Reduction. Cardiovasc Drugs Ther. 2021.

Publisher's Note Springer Nature remains neutral with regard to jurisdictional claims in published maps and institutional affiliations. 